\title{
SUICIDAL CUT THROAT INJURY AND SUCCESSFUL REPAIR
}

Sangeeta Aggarwal1, Barjinder Singh Sohal²

${ }_{1}^{1}$ Assistant Professor, Department of ENT, GMC \& H, Patiala, Punjab, India.

${ }^{2}$ Senior ENT Consultant, Patiala Heart Institute, Patiala, Punjab, India.

HOW TO CITE THIS ARTICLE: Aggarwal S, Sohal BS. Suicidal cut throat injury and successful repair. J. Evolution Med. Dent. Sci. 2019;8(08):544--546, DOI: 10.14260/jemds/2019/120

\section{PRESENTATION OF CASE}

A 45-year-old male from a rural area with multiple familial problems was brought to our emergency department by the local police with alleged history of self-inflicted cut throat injury within an hour of the incident. His clothes were blood stained. The patient was calm, conscious and cooperative. His B.P. was $90 / 60$ and pulse $110 / \mathrm{min}$ at the time of examination. He was not in respiratory distress on sitting up, but he became dyspnoeic on lying down. He was given intravenous fluids and I/V antibiotics on admission and blood transfusion was started.
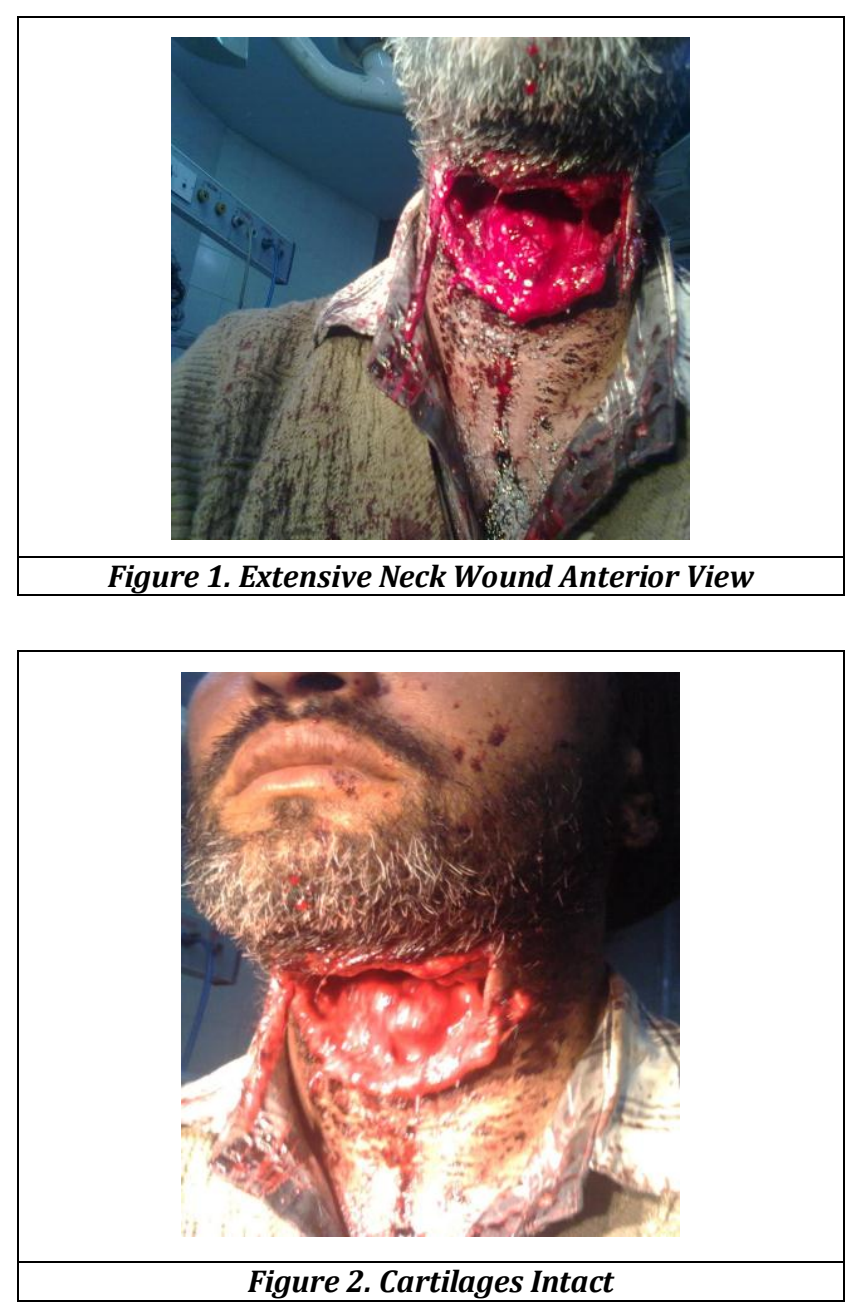

'Financial or Other Competing Interest': None.

Submission 28-12-2018, Peer Review 08-02-2019,

Acceptance 14-02-2019, Published 25-02-2019.

Corresponding Author:

Dr. Sangeeta Aggarwal,

The Art of Living Center,

147 Punjabi Bagh,

Patiala-147001, Punjab, India.

E-mail: aggarwalsangeetadr@gmail.com

DOI: $10.14260 /$ jemds $/ 2019 / 120$

(c) (i) $(9)$
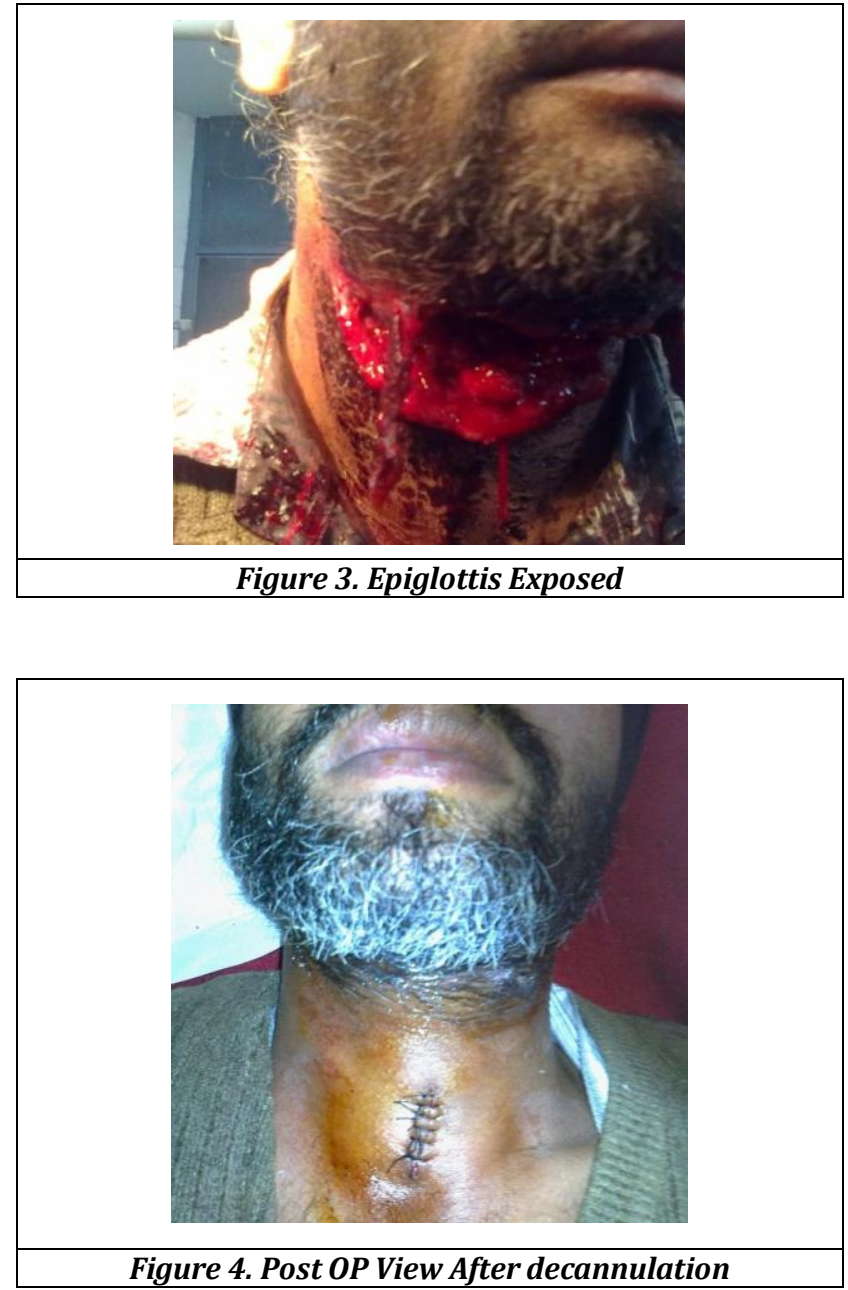

\section{CLINICAL DIAGNOSIS}

$10 \mathrm{cms} \times 4 \mathrm{cms}$ incised wound in the upper part of the neck above the thyroid cartilage with profuse salivary discharge and fresh bleeding from the wound site without injury to any major blood vessel. (Fig. 1), (Fig. 2), (Fig. 3).

\section{DIFFERENTIAL DIAGNOSIS}

a. Accidental Injury.

b. Homicidal Injury.

\section{PATHOLOGICAL DISCUSSION}

Cut-throat injury patients may present with airway compromise, aspiration, acute blood loss with hypoxemia if major blood vessels are involved. They pose initial management challenges in the casualty and for the anaesthesiologists in securing the airway. They may be fatal if timely intervention is delayed. Wound may be penetrating or non-penetrating, superficial or deep, involving soft tissues, cartilage, bones and neurovascular bundles or in combination.[1] 
Incised wounds of neck can be accidental, homicidal or suicidal whereas lacerated wounds are rarely suicidal in nature. Suicidal and homicidal injuries to the neck are mostly caused by sharp force. Accidental ones are usually caused by foreign objects such as metal or glass so usually do not result in extensive collateral tissue damage.[2]

Evaluation and management are complicated due to dense concentration of vital, vascular, aerodigestive and nervous system structures. A good team consisting of anaesthetists and surgeons [ENT] is required to prevent catastrophic airway, vascular or neurologic sequelae. Injury to major vessels [e.g. the carotid or jugular vessels] may be fatal.[1]

\section{DISCUSSION OF MANAGEMENT}

The patient was shifted to the emergency OT where the anaesthetists examined him and there was confusion on how to intubate him which caused some delay in the repair of the neck wound. First tracheostomy was planned under local anaesthesia, but when the patient was asked to lie down, he became dyspnoeic and restless. So, the mucosa of the laryngopharynx was anaesthetised with local spray and the endotracheal tube was introduced by lifting the thyroid cartilage via the open neck wound. Oxygenation was started, and after securing the airway, tracheostomy was done under local anaesthesia to maintain air passage away from the wound site. Then general anaesthesia was given through the tracheostomy tube and detailed examination of the wound was done on the table. The cut was infrahyoid at the level of thyrohyoid membrane. It was involving the strap muscles of both the sides and both the sternocleidomastoids. The epiglottis, thyroid cartilage and cricoid cartilages were intact. Pyriform fossae on both the sides were visible. The hyoid bone was also intact. The submandibular gland on left side was exposed and injured.

The wound was washed and cleaned with normal saline. Naso-gastric tube was inserted for post-operative feeding, before repair of the wound. The wound was repaired in layers after flexing the cervical spine. First the pharyngeal mucosa, then all the strap muscles, fascia and platysma were repaired with cat gut. The submandibular gland was stitched and left in situ. The skin was repaired with 3-0' Mersilk and drains put on either side of the wound and anti-septic dressing (ASD) was done. The drains were removed on 3 rd postop day and stitches were removed by $10^{\text {th }}$ postop day. The patient started taking orally semi-solids by $9^{\text {th }}$ day and $\mathrm{N} / \mathrm{G}$ tube was removed on $10^{\text {th }}$ day. Decannulation was done on $11^{\text {th }}$ day. (Fig. 4)

Post-operatively, the neck of the patient was kept slightly flexed to prevent undue tension on the stitches. In addition to the I/v fluids, the initial feeding was given through the $N / G$ tube. Post-operative period was uneventful, except there was minimal leakage of saliva from left side of the wound, maybe due to injury to the submandibular gland. Due to medicolegal problems the patient was discharged on the $12^{\text {th }}$ postop day with normal phonation and tolerating oral semi-solid and liquid diet.

\section{FINAL DIAGNOSIS}

Suicidal cut-throat injuries are either unreported or rare in our country. The management of these injuries requires a multidisciplinary approach. The purpose of this report is to emphasize that suicidal cut-throat injuries do occur in our society and there is a need for collaboration of the otolaryngologist, anaesthetist, and psychiatrist in the effective management of these patients. Ways must be found to identify people in society without mental disorders who are at risk of suicidal behaviours. Suicide is a known worldwide leading cause of death with psychiatric illnesses listed among the strongest predictors. Other predictors listed are familial troubles and poverty.[3] The patient who presented to us was divorced for a long time, had no children and was involved in extra-marital affairs, the later one being the cause of his suicidal cut-throat injury.

For effective management of a patient with cut-throat injury first the air-way should be properly maintained and haemostasis secured to prevent its aspiration. Also, the airway management should not be delayed excessively by radiological studies since an apparently stable airway may rapidly progress to an acute airway obstruction. Many patients with upper airway injuries may be successfully managed using traditional techniques to establish an airwayintubation through an obvious defect or endotracheal/orotracheal intubation or tracheostomy.[4]

Being a very rare case at our institution there was some delay in achieving the proper method of administering general anaesthesia. But once tracheostomy was done under local anaesthesia and mild sedation, the management of the airway was well-executed and repair of the wound in layers was achieved.

For surgical repair of such wounds, anatomically, the neck can be divided into three major zones: zone 1 below cricoid to thoracic inlet, zone 2 from cricoid to angle of mandible, and zone 3 above angle of mandible.[5] Under this division, our case can be classified into zone 2 .

The leading cause of death from neck injuries is haemorrhage from vascular structures.[3] There was no injury in our patient to any major blood-vessels or deep structures of the neck especially trachea, oesophagus or vertebral spine. The possible mechanism for carotid sparing would be the backwardly thrown head at the time of injury which is likely to draw the carotid sheath backwards.[4]

Venkatachalam et al have stressed that postop care includes fluid balance to prevent dehydration, humidification and aspiration of tracheobronchial secretions, cuff-pressure monitoring and broad spectrum antibiotics. [4]

Securing the airway should be the first priority and for this the patient may have to undergo cricothyroidotomy or urgent tracheostomy. Haemodynamic stabilization is required to treat the blood loss. Surgical repair is also a priority and to end without complication. These patients belong to lower socio-economic strata and have a lot of psycho-social problems which require proper counselling and follow up to avoid further accidents or incidents.

\section{REFERENCES}

[1] Kumar R, Madhusudhan R, Kumar K, et al. Paary: cut throat injuries - a challenge for airway and anaesthetic management - case report of 5 cases. Int J Cur Sci Res 2011;1(3):137-8.

[2] Naik S, Atal D, Murari A, et al. Cut throat injury by vehicular accident. IJMS 2012;3(1). 
[3] Adoga AA, Ma'an ND, Embu HY, et al. Management of suicidal cut throat injuries in a developing nation: three case reports. Cases Journal 2010;3:65.

[4] Venkatachalam SG, Selvaraj PDA, Rangarajan M, et al. An unusual case of penetrating tracheal ("Cut throat") injury due to chain snatching: The ideal airway management. India J Crit Care Med 2007;11(3):151-4.
[5] Darlong LM, Shunyu NB, Das R, et al. Cut throat zone II neck injury and advantage of a feeding jejunostomy. J Emerg Trauma Shock 2009;2(3):213-5. 